\title{
Referral to dentist in clinical ENT practice
}

\begin{abstract}
Usually ear related pain that presents in ENT clinic is pretty straightforward to diagnose and treat. But sometimes specialist see patients that are in pain that feels like it's in their ear, but it's actually not. The most essential component of a diagnosis is getting a clear history and doing clinical examination. If all regular tests fail to diagnose the problem then other potential causes of ear pain should be considered. Among several problems, referred pain originating from dental and associated structure is one of most common cause resulting in ear pain. Hence interdisciplinary approach with referral to dentist becomes very important in ruling out the possible source of dental pain that gets referred to ear.
\end{abstract}

Keywords: otogenic otalgia, referred otalgia, dental pain, temporomandibular joint disorder
Volume 6 Issue I - 2017

\author{
Sameer Anil Zope,' Girish Suragimath,' \\ Kumar Nilesh ${ }^{2}$ \\ 'Department of Period ontology Krishna University of Medical \\ Sciences University, India \\ ${ }^{2}$ Department of Oral and Maxillofacial Surgery Krishna \\ University of Medical Sciences University, India
}

Correspondence: Sameer Anil Zope Department of Period ontology Krishna University of Medical Sciences University Karad Maharashtra, India, Tel +919890669053,

Email aoldentist@gmail.com

Received: January 29, 2017| Published: February 20, 2017

\section{Introduction}

Ear pain, also known as earache or otalgia, is pain in the ear. The pain that originates inside the ear is called as Primary ear pain or otogenic otalgia. It can be caused by disease in the inner, middle, or external ear, but the three are difficult to distinguish in terms of the pain experienced..$^{1}$ If ear pain arises from pathologies in surrounding structures it is called as secondary or referred otalgia. 50 percent of secondary otalgia results from dental causes. ${ }^{2}$

Earache is not always associated with ear disease. Many patients who present with otalgia reveal no otological abnormalities. In such cases, distant secondary source of pain must be considered. ${ }^{3}$ Apart from problems within the ear itself, pain from the ears can be caused by impacted teeth, dental infections, neuralgia, sinus infections, temporomandibular joint dysfunction, and myalgia of masticatory muscles along with other conditions. Each of these conditions is associated with dental structures such as the teeth and jaws. ${ }^{4}$ These conditions can be easily ignored, resulting in needless and expensive investigations.

Referred earache can represent diagnostic challenge to ENT specialist. Making an accurate diagnosis depends on thorough understanding of complex anatomic relationship of ear and surrounding structures and a structured approach to patient evaluation which include referral to dentists.

\section{The neuroanatomic basis of referred otalgias}

The neuroanatomic basis of referred otalgias originating from dental and related pathologies rests within one of three general neural pathways. ${ }^{5}$ Hence, pathology in dental and associated structure of the body innervated by these neural pathways may refer pain to the ear. These general pathways are:

a. Via Trigeminal nerve cranial nerve V.

b. Via Facial nerve cranial nerve VII.

c. Via Glossopharyngeal nerve cranial nerve IX.

Potential dental causes for secondary otalgia

If you do all of your regular clinical investigations and find yourself still scratching your head trying to figure things out, consider these other potential dental causes for otalgia. ${ }^{2}$

\section{Acute dentoalveolar abscess}

Acute dent alveolar abscess, a condition which is characterized by accumulation of pus in tooth supporting structures, is typically accompanied by severe pain. Often that pain will radiate along the course of the nerve that innervate to the affected tooth. If the involved tooth is located in the posterior region of either jaw, the associated pain may refer to ear through trigeminal nerve pathway.

\section{Temporomandibular joint disorders}

Temporomandibular disorders are characterized by pain and dysfunction in temporomandibular joint and muscles that control its movement. As the TMJ is in very close vicinity of auditory meatus, it can be very difficult to distinguish whether pain is arising in the joints or in the ear. Pain may radiate to ear through auriculotemporal and massetric branch of mandibular nerve (branch of trigeminal nerve).

\section{Mayofascial pain, muscle spasm, myalgia of masticatory muscles}

Painful jaw muscles secondary to bruxism, space infections, and TMJ disorders may also transmit pain along nerve pathways that are difficult to differentiate from ear pain.

\section{Oral and oropharygeal carcinomas}

Carcinomas involving Oral cavity and oropharynx (base of tongue, soft palate, pharyngeal wall, tonsils) can also cause referred ear pain via trigeminal and glossopharyngeal nerve pathway.

\section{Parotid gland disorders}

Inflammatory, obstructive conditions and tumors of parotid gland may result in referred pain to ears through innervating glossopharyngeal and auriculotemporal nerve pathway.

\section{Conclusion}

Following referral from ENT specialist, dentist can play very important role in diagnosing the dental problems causing secondary otalgia. Moreover treating the dental cause will make this interdisciplinary approach more fruitful in ameliorating the ear pain. 


\section{Acknowledgments}

None.

\section{Conflicts of interest}

Author declares there are no conflicts of interest.

\section{Funding}

None.

\section{References}

1. Neilan RE, Roland PS. Otalgia. Med Clin North Am. 2010;94(5):961-971.
2. Ely JW, Hansen MR, Clark EC. Diagnosis of ear pain. Am Fam Physician. 2008;77(5):621-628.

3. Charlette SD, Coatesworth AP. Referred otalgia: a structured approach to diagnosis and treatment. Int J Clin Pract. 2007;61(6):1015-1021.

4. Kim DS, Cheang P, Dover S, et al. Dental otalgia. J Laryngol Otol. 2007;121(12):1129-1134.

5. Scarbrough TJ, Day TA, Williams TE, et al. Referred otalgia in head and neck cancer: a unifying schema. Am J Clin Oncol. 2003;26(5):e157e162. 